\title{
Cell cycle status in AML blast cells from peripheral blood, bone marrow aspirates and trephines and implications for biological studies and treatment
}

Rob S. Sellar ${ }^{1}$, Laura Fraser ${ }^{1}$, Asim Khwaja ${ }^{1}$, Rosemary E. Gale ${ }^{1}$, Teresa Marafioti2,3, Ayse Akarca ${ }^{2,3}$, Mike Hubank4, Tony Brooks4, Kai Stoeber2,, Gareth Williams², and David C. Linch¹.

Running title: $\mathrm{G}_{1}$-arrested blasts in AML bone marrow aspirates

Affiliations: ${ }^{1}$ Department of Haematology, University College London Cancer Institute, London, UK; 2Department of Histopathology, University College London Hospitals, London, UK; ${ }^{3}$ Department of Cellular Pathology, University College London Hospitals, London, UK; ${ }^{4}$ Genetics and Genomic Medicine Programme, UCL Institute of Child Health, London UK.

\author{
Corresponding Author: David C Linch \\ Department of Haematology, UCL Cancer Institute, \\ Paul O'Gorman Building, 72 Huntley Street, \\ London WC1E 6DD, UK \\ Tel: +44-20-7679-6226 \\ Fax: +44-20-7679-6222 \\ e-mail: d.linch@ucl.ac.uk
}




\section{Summary:}

Using immunohistochemistry and flow cytometry to define phases of the cell cycle this study shows that, a high proportion of AML blasts obtained from trephine biopsies are cycling whereas, $>95 \%$ of peripheral blood-derived blasts are arrested in $\mathrm{G}_{1}$. Furthermore, results obtained from bone marrow aspirates are more similar to those from blood rather than from the trephine biopsies, and these differences were confirmed by gene expression profiling in a patient with high count AML. This has implications for cell cycle and other biological studies using aspirates rather than trephine biopsies and for the use of cell mobilizing agents before chemotherapy. 
In acute myeloid leukaemia (AML), leukaemic blast cells from bone marrow (BM) and peripheral blood (PB) are generally assumed to be biologically similar, but previous studies of cell proliferation have suggested differences (Hiddemann et al, 1982a; Vidriales et al, 1995). It is not clear whether these relate to intrinsic differences in the blast cell populations or a higher proportion of non-leukemic quiescent cells in PB. Most studies of BM use aspirates (BMA), although it has long been recognized that they can be variably 'contaminated' by blood cells (Holdrinet et al, 1980; Hiddemann et al, 1982b). Furthermore, earlier studies of the cell cycle were limited by the need for in vitro culture to measure ${ }^{3} \mathrm{H}$-thymidine uptake by the proliferating cells, which was potentially subject to the influence of different accessory cell populations in PB and BM (Raza et al, 1990). With the availability of monoclonal antibodies against antigens present at different phases of the cell cycle, it is now possible, however, to determine the proliferative status of cells directly ex-vivo without in vitro culture (Williams and Stoeber, 2007). We have therefore used immunohistochemistry to determine the cell cycle status of AML blasts in BM using trephine biopsies (BMT), which are not subject to blood contamination, and compared this finding to the findings in BMAs and PB.

\section{Methods}

Full experimental details are provided in the Supplementary material. The UCL/UCLH Research Ethics Committee approved the studies. Informed consent was obtained according to the Declaration of Helsinki.

Analysis of cell cycle status of blast cells

For immunohistochemistry, serial sections from paraffin-embedded BMT and buffy-coat cell pellets from BMA and PB samples were immuno-stained with monoclonal antibodies to $\mathrm{Mcm} 2$ to assess nonquiescent cells (positive in $\mathrm{G}_{1}, \mathrm{~S}, \mathrm{G}_{2}$ and $\mathrm{M}$ but not $\mathrm{G}_{0}$ ), MIB-1 (Ki-67) for actively proliferating cells (positive in $\mathrm{G}_{1}, \mathrm{~S}, \mathrm{G}_{2}$ and $\mathrm{M}$ but not $\mathrm{G}_{0}$ ), and Geminin for cycling cells that have progressed beyond $\mathrm{G}_{1}$ (positive in $S / G_{2} / M$ but not $G_{1}$ and $G_{0}$ )(Williams and Stoeber, 2007). For each section a minimum of 200 blasts were assessed and labeling indexes (LI) determined from the number of positive nuclearstained cells as a percentage of the total number of cells. For flow cytometry, the percentage of blasts in $S / G_{2} / M$ phases of the cell cycle was assessed using propidium iodide staining of fixed cells. 
Determination of peripheral blood-derived cells in bone marrow aspirates from haematologically normal individuals

BMT, BMA and PB samples were collected from 13 individuals with a morphologically normal BM undergoing a BM harvest. The BMA samples (5-10mLs) were collected and paired with 10mLs of blood. Core biopsies were obtained from the posterior iliac crest and the length of the biopsy determined using a vernier caliper. The biopsies were then teased apart, single cell suspensions prepared, and the number of cells counted. Actual volumes of the collected BMT biopsies were calculated from their measured length and the internal cross sectional diameter of the Jamshidi needles used $\left(4.486 \mathrm{~mm}^{2}\right)$. Cytospins were prepared from the BMT, BMA and PB samples, stained with May-Grunwald-Giemsa, and manual differential counts performed. The volumetric contributions of BMT and PB to the BMA was then calculated as described with an example in the legend to Table 1.

RNA expression profiling

An mRNA-focused library was prepared using total RNA from BMT, BMA and PB samples processed within 30 minutes of collection from a patient with high count AML and then sequenced (Supplementary material). De-multiplexed FastQ data was processed and normalized counts (FPKM) were generated and visualized in GeneSpring 13.0 (Agilent Technologies).

\section{Results}

Cell cycle analysis of blast cells in peripheral blood, bone marrow aspirates, and bone marrow biopsies

Matched BMT, BMA and PB samples were obtained from eight AML patients prior to therapy. Median age was 64 years (range, 34-74); median peripheral white cell count (WCC) was $27.5 \times 10^{9} / \mathrm{L}$ (range, 2365). There was no significant difference between BMT and PB samples in the proportion of blasts expressing Mcm2 ( $P=.17)$ (Figure 1A, Figure S1A), indicating that nearly all the blast cells were nonquiescent, irrespective of location. The percentage of blasts in-cycle was also highly correlated between BMT and PB ( $\mathrm{r}=.98)$. There was, however, a significant difference in the proportion of blasts expressing MIB-1, median LI 63\% for BMT versus 4\% for PB ( $P=.008)$ (Figure 1A, Figure S1B), indicating that the BMT blasts had a very high proliferative fraction whereas, in general, PB blasts were not actively proliferating. In accord with this, the median Geminin LI indicating progression beyond G1 was $24 \%$ for BMT versus $1 \%$ for PB $(P=.008)$ (Figure $1 \mathrm{~A}$, Figure S1C). The MIB-1 and Geminin LIs for BMAs were more akin to those of PB rather than BMT blasts, suggesting that large volume BMAs may be heavily contaminated with blood cells. Data from flow cytometric cell-cycle 
analysis showed good correlation between the proportion of geminin-positive and propidium iodidestained cells in $S / G_{2} / M$, irrespective of location ( $r=.986$ ) (Figure S2, Figure S3).

\section{Determination of peripheral blood derived cells in bone marrow aspirates}

To further assess the impact of blood contamination on BMAs, matched BMT, BMA and PB samples from 13 haematologically normal individuals were studied. In the BMTs, nucleated red cells comprised a median $28 \%$ of total nucleated cells (range, $11 \%-59 \%$ ) compared to $0 \%$ in PB and $17 \%$ (range, 5\%$25 \%$ ) in BMAs (Table 1). Overall, therefore, a median 53\% of the BMA cells were BM-derived and $47 \%$ PB-derived. The volumetric contribution of BM and PB in the BMAs required to achieve these proportions is dependent on the cell concentrations. The median BMT nucleated cell count was $91.6 \times 10^{9} / \mathrm{L}$, which is $\approx 16$ fold higher than the median WCC of $5.9 \times 10^{9} / \mathrm{L}$ in normal individuals (Simmons et al, 1974). Using this ratio and the median cellular proportions described above, it can be estimated that, by volume, $93 \%$ of a BMA is from PB (Table 1). If the same volumetric contribution of PB to a BMA also pertains to leukemic samples, it can be speculated that a BMT count of $250 \times 10^{9} / \mathrm{L}$ $\left(\approx 2.5 \times\right.$ normal BM cellularity) and PB WCC of $10 \times 10^{9} / \mathrm{L}, 35 \%$ of the BMA nucleated cells will be PBderived cells, but if the PB WCC is $100 \times 10^{9} / \mathrm{L}$, this proportion rises to $84 \%$.

Comparison of peripheral blood, bone marrow aspirate, and bone marrow biopsy by gene expression profiling

Gene expression profiling of BMT, BMA and PB samples was carried out from a patient with a presenting WCC of $98 \times 10^{9} / \mathrm{L}$ and $>90 \%$ blasts in all three samples, precluding any need for blast cell purification. Evaluable data was obtained from 10,363 genes. There were 472 non-stromal genes expressed at $\geq 2$ fold higher levels in the BMT than PB, of which 146 were related to the cell-cycle, including members of the Mcm family, Geminin, and MKI67 encoding Ki-67 (Table S2). The cell-cycle expression profile of the BMA was clearly more akin to that of PB than BMT (Figure 1B).

\section{Discussion}

These data demonstrate that the majority of blasts in peripheral blood exist in a $\mathrm{G}_{1}$ arrested state and are not actively proliferating. This is in marked contrast to blast cells obtained from biopsies where although a similar number of blasts are in-cycle as defined by $\mathrm{Mcm} 2$ expression, a far greater number are actively proliferating and have progressed beyond $G_{1}$ into $S / G_{2} / M$. Furthermore, we demonstrate that BMAs are heavily contaminated with PB derived blasts when the PB cell count is high and if cell proliferation studies are performed on BMA, paradoxically, the cell proliferation rates would appear lower in those cases with the highest peripheral counts. This might account for some of the reported 
inconsistencies on the impact of cell-cycle status on outcome in AML (Hiddemann et al, 1982a; Preisler et al, 1984; Raza et al, 1990; Raza et al, 1991; Vidriales et al, 1995; Braess et al, 2001). The differences may be less pronounced if small volume aspirates are taken, but this is often not the case when cells are required for immune-phenotyping, cytogenetics, molecular analyses and bio-banking. This indicates that studies of cell cycle analysis in AML must use BMTs and not be based on the findings in BMAs.

These findings are supported by the gene expression profiling of matched BMT, PB, and BMA samples from a patient with AML, where the gene expression of BMT blasts is shown to be distinct from BMA blasts which are very similar to PB blasts. A large proportion of the discrepancies between BMT and BMA/PB samples were genes with a role in the cell cycle but other genes were also differentially expressed including the down-regulation of 17 transcription factors in the BMA/PB samples. This suggests another level of complexity in the interpretation of BMA gene expression profiling to ascertain prognosis in AML and to understand the biology of normal haematopoiesis.

Finally, these studies suggest that circulating blasts will be less susceptible to treatment with cell-cycle phase specific agents such as cytosine arabinoside, the mainstay of current AML therapy. Nevertheless, by virtue of not having entered quiescence, these cells may be capable of rapid proliferation if they recirculate back to the BM. This is particularly relevant to the potential use of CXCR4 inhibitors to mobilize blast cells into the circulation prior to the administration of chemotherapy (Nervi et al, 2009). 


\section{Acknowledgements}

This work was supported by Cancer Research UK, Bloodwise and the UCLH/UCL NIHR Biomedical Research Centre.

\section{Authorship contributions}

R.S.S., L.F., K.S., G.W. and D.C.L. designed the study; R.S.S, L.F., T.M., A.A. and T.B. performed

experimental work; R.S.S., A.K., R.E.G., M.H. and D.C.L. analyzed data; R.S.S., A.K., R.E.G., M.H. and D.C.L. wrote the manuscript, which was reviewed by all authors.

\section{Conflicts of interest}

The authors declare no competing conflicts of interest. 


\section{References}

Braess, J., Jahns-Streubel, G., Schoch, C., Haase, D., Haferlach, T., Fiegl, M., Voss, S., Kern, W., Schleyer, E. \& Hiddemann, W. (2001) Proliferative activity of leukaemic blasts and cytosine arabinoside pharmacodynamics are associated with cytogenetically defined prognostic subgroups in acute myeloid leukaemia. Br J Haematol, 113, 975-982.

Hiddemann, W., Buchner, T., Andreeff, M., Wormann, B., Melamed, M.R. \& Clarkson, B.D. (1982a) Cell kinetics in acute leukemia: a critical reevaluation based on new data. Cancer, 50, 250-258.

Hiddemann, W., Buchner, T., Andreeff, M., Wormann, B., Melamed, M.R. \& Clarkson, B.D. (1982b) Bone marrow biopsy instead of 'marrow juice' for cell kinetic analysis. Comparison of bone marrow biopsy and aspiration material. Leuk Res, 6, 601-612.

Holdrinet, R.S., von Egmond, J., Wessels, J.M. \& Haanen, C. (1980) A method for quantification of peripheral blood admixture in bone marrow aspirates. Exp Hematol, 8, 103-107.

Nervi, B., Ramirez, P., Rettig, M.P., Uy, G.L., Holt, M.S., Ritchey, J.K., Prior, J.L., Piwnica-Worms, D., Bridger, G., Ley, T.J. \& DiPersio, J.F. (2009) Chemosensitization of acute myeloid leukemia (AML) following mobilization by the CXCR4 antagonist AMD3100. Blood, 113, 6206-6214.

Preisler, H.D., Azarnia, N., Raza, A., Grunwald, H., Vogler, R., Browman, G., Goldberg, J., Chervenick, P., Miller, K., Brennan, J. \& et al. (1984) Relationship between the per cent of marrow cells in $S$ phase and the outcome of remission-induction therapy for acute nonlymphocytic leukaemia. Br J Haematol, 56, 399-407.

Raza, A., Preisler, H.D., Day, R., Yasin, Z., White, M., Lykins, J., Kukla, C., Barcos, M., Bennett, J., Browman, G. \& et al. (1990) Direct relationship between remission duration in acute myeloid leukemia and cell cycle kinetics: a leukemia intergroup study. Blood, 76, 21912197.

Raza, A., Preisler, H., Lampkin, B., Yousuf, N., Tucker, C., Peters, N., White, M., Kukla, C., Gartside, P., Siegrist, C. \& et al. (1991) Biological significance of cell cycle kinetics in 128 standard risk newly diagnosed patients with acute myelocytic leukemia. $\mathrm{Br} J$ Haematol, 79, 33-39.

Simmons, A., Leaverton, P. \& Elbert, G. (1974) Normal laboratory values for differential white cell counts established by manual and automated cytochemical methods (Hemalog DTM). J Clin Pathol, 27, 55-58.

Vidriales, M.B., Orfao, A., Lopez-Berges, M.C., Gonzalez, M., Lopez-Macedo, A., Ciudad, J., Lopez, A., Garcia, M.A., Hernandez, J., Borrego, D. \& et al. (1995) Prognostic value of S-phase cells in AML patients. Br J Haematol, 89, 342-348.

Williams, G.H. \& Stoeber, K. (2007) Cell cycle markers in clinical oncology. Curr Opin Cell Biol, 19, 672-679. 


\section{Figure legend}

Figure 1. Comparison of blast cells from matched bone marrow trephine, bone marrow aspirate and peripheral blood samples from AML patients. (A) Relative proportion of blasts that were licenced for replication as defined by Mcm2 staining, were actively cycling as defined by MIB-1 staining, and had progressed beyond $G_{1}$ into $S / G_{2} / M$ phase as defined by Geminin staining. (B) Heat map showing RNA expression analysis of 149 cell cycle-related genes with $>2$ fold difference in expression between BMT and PB samples from an AML patient. Of these, 146 were down-regulated and 3 were up-regulated in PB compared to the BMT. The colour range refers to the median normalized $\log _{\mathrm{e}} 2$ values across samples. LI, labelling index (number of positive cells as a percentage of total number of positive and negative cells); IHC, immunohistochemistry. 
Table 1. Derivation of the proportion of BM- and PB-derived cells in bone marrow aspirate samples from 13 hematologically normal individuals and calculation of the median volumetric contribution of PB in the aspirates.

\begin{tabular}{|l|c|c|c|c|c|c|}
\hline No. & \multicolumn{2}{|c|}{ BMT } & PB & \multicolumn{2}{c|}{ BMA } \\
\hline & $\begin{array}{c}\text { Nucleated } \\
\text { cell count } \\
(\mathrm{x} \mathrm{10} / \mathrm{L})\end{array}$ & $\begin{array}{c}\% \text { nucleated } \\
\text { red cells }\end{array}$ & $\begin{array}{c}\% \text { nucleated } \\
\text { red cells }\end{array}$ & $\begin{array}{c}\% \text { nucleated } \\
\text { red cells }\end{array}$ & $\begin{array}{c}\text { Proportion of BM- } \\
\text { derived cells* }\end{array}$ & $\begin{array}{c}\text { Proportion of } \\
\text { PB-derived cells* }\end{array}$ \\
\hline 1 & 118.0 & $12 \%$ & $0 \%$ & $5 \%$ & $42 \%$ & $58 \%$ \\
\hline 2 & 84.4 & $20 \%$ & $0 \%$ & $20.5 \%$ & $100 \%$ & $0 \%$ \\
\hline 3 & 165.0 & $45 \%$ & $0 \%$ & $24 \%$ & $53 \%$ & $47 \%$ \\
\hline 4 & 91.6 & $23 \%$ & $0 \%$ & $10 \%$ & $43 \%$ & $57 \%$ \\
\hline 5 & 187.0 & $59 \%$ & $0 \%$ & $11 \%$ & $19 \%$ & $81 \%$ \\
\hline 6 & 55.0 & $24 \%$ & $0 \%$ & $23 \%$ & $96 \%$ & $4 \%$ \\
\hline 7 & 68.0 & $32 \%$ & $0 \%$ & $15 \%$ & $47 \%$ & $53 \%$ \\
\hline 8 & 374.0 & $36 \%$ & $0 \%$ & $25 \%$ & $69 \%$ & $31 \%$ \\
\hline 9 & 130.5 & $33 \%$ & $0 \%$ & $16.5 \%$ & $50 \%$ & $50 \%$ \\
\hline 10 & 79.1 & $19 \%$ & $0 \%$ & $15.5 \%$ & $82 \%$ & $18 \%$ \\
\hline 11 & 85.0 & $28 \%$ & $0 \%$ & $20 \%$ & $71 \%$ & $29 \%$ \\
\hline 12 & 88.9 & $31 \%$ & $0 \%$ & $16.5 \%$ & $53 \%$ & $47 \%$ \\
\hline 13 & 158.0 & $11 \%$ & $0 \%$ & $17 \%$ & $100 \%$ & $0 \%$ \\
\hline Median & 91.6 & $28 \%$ & $0 \%$ & $17 \%$ & $53 \%$ & $47 \%$ \\
(range) & $(55-374)$ & $(11-59 \%)$ & & $(5-25 \%)$ & $(19-100 \%)$ & $(0-81 \%)$ \\
\hline
\end{tabular}

*The proportion of BMT- and PB-derived cells in the BMA samples has been calculated from the \% nucleated red cells in the BMT, PB and BMA samples from each individual. As the $\%$ of nucleated red cells in the blood is in all cases zero, the \% nucleated red blood cells in the BMA is the \% nucleated red blood cells in the BMT $\mathrm{x}$ the fraction of the BMA composed of the BMT by volume. For example, for individual 1, the BMA had 5\% nucleated red cells, which is 12\%(\% nucleated red cells in the trephine), multiplied by $\mathrm{x}$ where $\mathrm{x}$ is the fraction of BMA contributed by the BMT. Therefore $\mathrm{x}=5 / 12=0.42=$ $42 \%$ and therefore $58 \%$ of the cells must have come from the PB.

Abbreviations: BM, bone marrow; BMA, bone marrow aspirate; BMT, bone marrow trephine; PB, peripheral blood. 